\title{
Protocol
}

\section{Imaging Cell Movements in Egg-Cylinder Stage Mouse Embryos}

\author{
Shankar Srinivas
}

\section{INTRODUCTION}

Cell movements in the pregastrulation egg cylinder mouse embryo play an important role in patterning. The stereotypic movement of the anterior visceral endoderm converts a proximal-distal axis to an anteroposterior axis by properly positioning the primitive streak. The epiblast at this stage is also characterized by a great deal of cell mixing, about which very little is known. Visualizing such cell movements can help us understand their role in embryonic development. This protocol describes a method to isolate and culture the egg cylinder-stage mouse embryo, as well as an approach for time-lapse imaging of embryos cultured in vivo.

\section{MATERIALS}

CAUTIONS AND RECIPES: Please see Appendices for appropriate handling of materials marked with $\langle!>$, and recipes for reagents marked with $\langle\mathbf{R}>$.

\section{Reagents}

Culture medium

This is a 1:1 mix of heat-inactivated mouse serum (from Step 11) and supplemented CMRL medium. DMEM can be used instead of CMRL, but CMRL gives more consistent results.

$<$ ! > Isoflurane

M2 medium (Sigma-Aldrich)

Mice

Mineral oil, embryo-tested (Sigma-Aldrich) (optional; see Step 22)

$<$ R $>$ Supplemented CMRL medium

\section{Equipment}

Dissection instruments

Dissection microscope, equipped with transmitted light illumination from below and a fiber optic cold light source for illumination from above

Environmental enclosure

Pregastrulation egg-cylinder stage mouse embryos are very sensitive to environmental conditions, particularly temperature fluctuations. Environmental enclosures that maintain the entire microscope at a set temperature are far preferable to stage-top environmental chambers; they generally maintain a more stable temperature and provide more flexibility in terms of the dish used to culture embryos. Embryos develop better in media that are not buffered with HEPES, and hence, the local atmosphere needs to be a humidified $5 \% \mathrm{CO}_{2} /$ air mix. This generally can be achieved easily by fashioning a small, clear, plastic box that can be placed on top of the culture dish and into which one can supply premixed $5 \% \mathrm{CO}_{2}$ /air that has been bubbled through warm water to humidify it.

Forceps, fine microdissection

Common forceps are acceptable, provided they have been sharpened on a polishing stone.

Adapted from Imaging in Developmental Biology (ed. Sharpe and

Wong). CHSL Press, Cold Spring Harbor, NY, USA, 2011 (in press).

Cite as: Cold Spring Harb Protoc; 2010; doi:10.1101/pdb.prot5539

www.cshprotocols.org 
Ice

Lab-Tek II eight-well rectangular chambers, cover glass-bottomed (Nalge Nunc International) MatTek glass-bottomed 35-mm culture dishes can be used as an alternative (see Step 21).

$<$ ! $>$ Liquid nitrogen

Micropipettor (e.g., P20 Pipetman) and tips

Microscope and analysis software

Because the embryo needs to be immersed in medium for culture, an inverted microscope is generally most suitable for imaging. Any compound microscope should be suitable as long as it is equipped with 10X, 20X, and/or $40 X$ objectives and a high-sensitivity camera (such as a cooled charge-coupled device [CCD] camera or a photomultiplier tube on a laser-scanning confocal microscope). Ideally, the microscope should also be equipped with automated shutters and control software to take time-lapse images automatically. An automated $x-y-z$ stage is not essential, but is a great advantage because it allows several embryos to be imaged over the course of one experiment.

Using just bright-field optics, one can discern cell outlines in the visceral endoderm, but epifluorescence microscopy is essential for more detailed imaging using fluorescently labeled embryos. Standard wide-field epifluorescence, laser scanning, and spinning disk confocal systems are all suitable, and each has its advantages and disadvantages. Confocal microscopes can provide higher-quality images than wide-field epifluorescence microscopes, but they can also be less sensitive, and hence, lead to more photodamage to the imaged embryo. However, highly sensitive laser-scanning confocal microscopes like the Zeiss 710 provide high-quality images with minimal photodamage. High-quality images can also be obtained with specialized wide-field epifluorescence microscopes such as the DeltaVision system (Applied Precision). Objectives such as the Zeiss CApochromat 40X/1.2 numerical aperture (NA) water-immersion lens are particularly suitable. Their high NA allows more light to be collected, and the resulting images they collect suffer fewer aberrations because the refractive index of the immersion medium (e.g., water or a water substitute) is close to that of the culture medium.

Needle, 25-gauge

Petri dishes, plastic, 35-mm

Pin vise for holding tungsten needles

Syringe, 1 -cc

Tissue culture incubator preset to $37^{\circ} \mathrm{C}$ and $5 \% \mathrm{CO}_{2}$

Tubes, microcentrifuge

Tungsten wire for making needles

For a method for sharpening tungsten needles, see Hogan et al. (1994).

Ultracentrifuge, benchtop

\section{METHOD}

\section{Preparation of Mouse Serum}

Beddington (1987) presents an alternative approach to preparing rat serum that is also applicable to the preparation of mouse serum.

1. Following a local Institutional Animal Care and Use Committee (IACUC)-approved procedure, anesthetize the mouse using an inhalant anesthesia (e.g., isoflurane). Kill the mouse by cervical dislocation.

Be careful to snap the neck cleanly, to avoid internal bleeding from torn blood vessels in the neck.

2. Immediately dissect open the thoracic cavity to expose the heart.

3. Insert a 25-gauge needle (attached to a 1-cc syringe) into the heart; generally, the right ventricle is easiest. Allow the blood to enter the syringe. Facilitate this by gently pulling on the plunger, but do not aspirate the blood by pulling vigorously because this will cause hemolysis.

4. Detach the needle from the syringe. Transfer the blood slowly to a microcentrifuge tube.

5. Immediately centrifuge the blood at room temperature at $15,000 \mathrm{~g}$ for $3 \mathrm{~min}$. This separates the plasma (top layer) from the cells (bottom layer).

6. Using a sterile pipette tip, collect the plasma. Transfer to a fresh microcentrifuge tube on ice. 
Once in the rhythm of things, you should be able to perform Step 6 for the previous sample and Steps 1-4 for the next sample, while centrifuging the current sample for $3 \mathrm{~min}$.

7. Incubate all the plasma samples on ice for 3-4 h, during which a fibrin clot will form. At this stage, visually screen the samples and discard those showing extensive hemolysis (i.e., a strong pink color).

8. Centrifuge the samples at $15,000 \mathrm{~g}$ for $10 \mathrm{~min}$ at $4{ }^{\circ} \mathrm{C}$ to pellet the fibrin clot and any residual cells. Collect the serum with a sterile pipette tip.

If the fibrin clot does not pellet, press it against the wall of the microcentrifuge tube with a pipette tip to collapse it, and then aspirate the serum.

9. Pool all the serum collected for the day to minimize variations among individual mice.

10. Aliquot the serum into microcentrifuge tubes $(500 \mu \mathrm{L}$ per aliquot is generally suitable). Flash-freeze in liquid nitrogen. Store at $-80^{\circ} \mathrm{C}$.

11. Prepare heat-inactivated serum:

i. Puncture the top of a microcentrifuge tube containing the mouse serum before inactivation to allow any dissolved inhalant anesthetic to escape.

ii. Heat-inactivate the serum for $30 \mathrm{~min}$ at $55^{\circ} \mathrm{C}$.

Heat-inactivated mouse serum can be used for $\sim 1 \mathrm{wk}$ if stored at $4^{\circ} \mathrm{C}$.

\section{Dissection of Pregastrulation Egg-Cylinder Stage Embryos}

The instructions below are guidelines. Try variations to identify what works best for you.

12. Dissect out the two uterine horns into a dish of room-temperature M2 medium in a 35-mm Petri dish.

Take care to remove as much attached fat as possible before placing the uterus in the dish; this will keep the medium clearer at later stages, making dissections easier.

13. Starting from any end of the uterus, dissect out the decidua one by one: Use a dissection microscope with illumination from above for the following steps.

i. Grasp the uterus to the left of the deciduum with one pair of forceps. Orient the uterus so that the smooth antimesometrial side is uppermost.

Make sure that the tip of the forceps extends just beyond the mesometrial extent of the uterus Fig. 1A) so that when orienting the uterus, it can be stabilized by resting the tip of the forceps on the floor of the plastic dish.

ii. Use the sharp tip of one arm of another forceps to puncture the antimesometrial uterine wall (Fig. 1B). Slide the forceps arm through the uterus and out the other side (Fig. 1C). When poking through the uterus with the forceps, try to span a generous segment of uterine wall, because this opens up a larger "window" and ensures that the subsequent step is very easy.

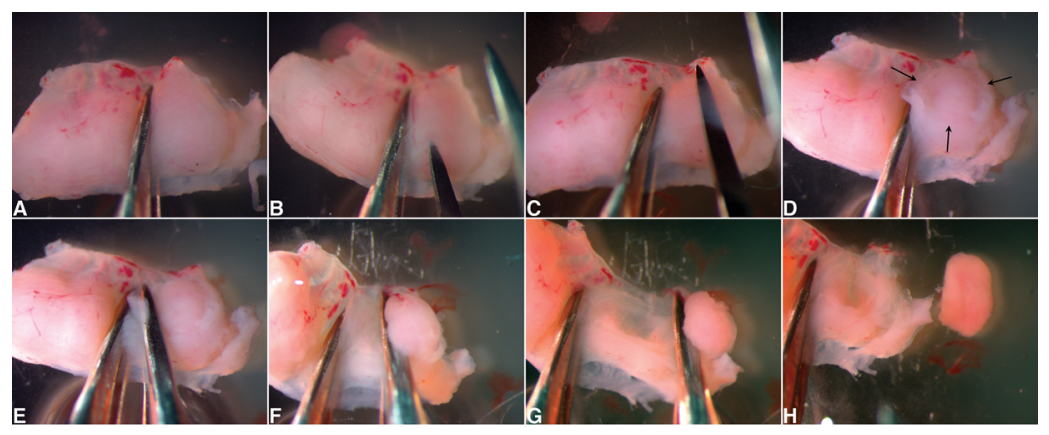

FIGURE 1. $(A-H)$ Sequence of steps for isolating embryonic day 5.5 (E5.5) deciduum from the uterus. The arrows in $D$ show the cut margins of the uterine wall, to indicate how large the opening should ideally be. For details, see Step 13. 
iii. Tear the uterine wall by pulling firmly on the forceps, in the direction of the long axis of the uterus.

This should open the uterine wall, exposing the deciduum (Fig. 1D).

iv. Very lightly grasp the uterus with half-closed forceps. Slide them along the uterus and beneath the deciduum, freeing it from the uterus (Fig. 1E-H).

v. Repeat Step 13.iv with the next deciduum along the uterus.

After removing two or three decidua, trim off the uterine tissue from which previous decidua have been removed, so that it does not get in the way.

14. Transfer the decidua to fresh room-temperature $M 2$ in the lid of the $35-\mathrm{mm}$ dish used to isolate the decidua.

The lid has shorter walls than the actual dish, making the subsequent dissection easier.

15. Dissect out the embryo from each deciduum as follows:

Switch off the illumination from above, and use only transmitted illumination from below for the following steps.

i. Orient the deciduum so that the end that has a midline groove (and is slightly blunter) is positioned to the right (Fig. 2A).

ii. Pin the deciduum down on the dish with a closed set of forceps pressed into the middle of the deciduum, near the inside end of the groove (Fig. 2B).

iii. Use another set of closed forceps to tear the right half of the deciduum open along the midline in a single motion (Fig. 2B,C).

Do not make many small cuts, because this will create jagged edges in the deciduum that make it difficult to locate the embryo.

iv. Open up the deciduum by inserting a closed forceps into the tear and allowing the forceps to open. At the same time, pivot the deciduum into a vertical position, with the torn half facing up, so that you can look down into its interior.

v. Pin the open deciduum down on the floor of the dish using forceps. Identify the embryo: It should be visible as a slight shadow, often located near a darkly pigmented structure in the deciduum.

Adjust the angle of the illumination if necessary to see the embryo clearly (Fig. 2D-F).

vi. Pinch into the decidual tissue beneath with forceps. Scoop out the embryo and move it to one side of the dish, so that it is out of the way while isolating embryos from the other decidua.

Embryos can be moved by gently swirling the medium around them with forceps, or by picking them up in a pipette tip. If using a pipette tip, make sure to aspirate $M 2$ medium into the tip once or twice before handling the embryo, because this helps prevent the embryo from sticking to the plastic wall of the tip.

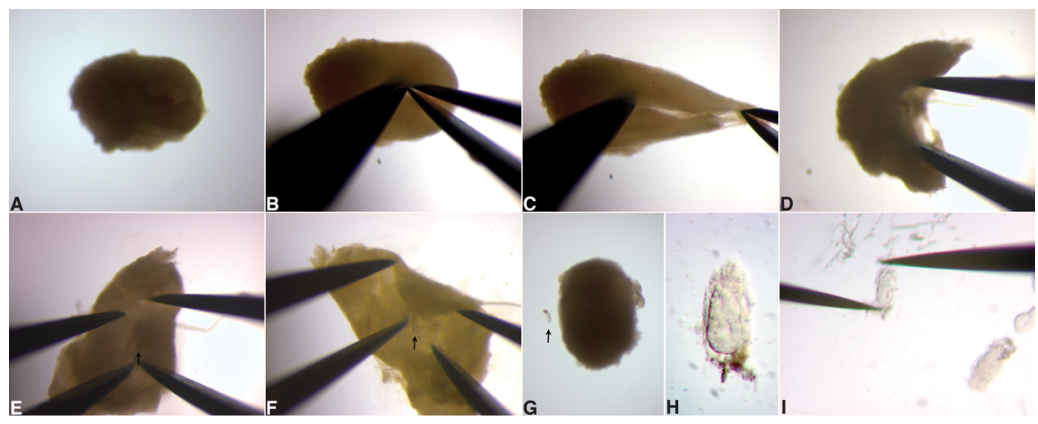

FIGURE 2. (A-F) Sequence of steps for isolating an E5.5 embryo from a deciduum. (G) An embryo and deciduum next to each other to provide a sense of scale. The embryo in this panel is $\sim 100 \mu \mathrm{m}$ in width. The arrow in $E$-G points to the embryo. $(H)$ An isolated E5.5 embryo, with intact Reichert's membrane. (I) Removal of the Reichert's membrane with tungsten needles. 
16. After all the embryos have been isolated, remove Reichert's membrane.

Continue using illumination from below on the dissection microscope. Using the following method, with a little practice one can become adept at removing Reichert's membrane quickly and without damage to the embryo. For an alternate approach to removing the membrane using micromanipulators, see Miura and Mishina (2003). For an enzymatic procedure, see Rivera-Perez et al. (2007).

i. Insert one tungsten needle into the space between Reichert's membrane and the embryo to pin down the embryo.

Generally, it is easiest to do this where Reichert's membrane meets the ectoplacental cone. Sometimes, there is also a fair amount of space at the distal tip between the embryo and Reichert's membrane (Fig. $2 \mathrm{H}, \mathrm{I})$.

ii. Use a second tungsten needle to peel off Reichert's membrane.

iii. Remove any Reichert's membrane attached to the embryo using the tungsten needles. When cutting with tungsten needles, hold the needles so that they are opposed and in contact with each other, forming an $X$ shape. To cut, draw the needles apart so that the point of contact between the needles travels toward the tip of the needles (in a manner similar to the action of scissors). This cuts the intervening Reichert's tissues neatly and avoids damaging the embryo.

17. Store embryos in $\mathrm{M} 2$ medium for up to $1-2 \mathrm{~h}$, preferably at $37^{\circ} \mathrm{C}$.

\section{Imaging Cell Movements in the Egg Cylinder Embryo}

18. Equilibrate culture medium for $2-3 \mathrm{~h}$ in a tissue culture incubator at $37^{\circ} \mathrm{C}$ and $5 \% \mathrm{CO}_{2}$. Medium can also be equilibrated overnight, but preferably for not more than $\sim 18 \mathrm{~h}$.

19. Equilibrate the microscope environmental chamber to $37^{\circ} \mathrm{C}$ for at least $3-4 \mathrm{~h}$, but preferably overnight.

Temperature stability is important for embryos to culture well, and some components of the microscope (e.g., the objectives) can take several hours to equilibrate.

20. Using a pipette tip, wash away excess $M 2$ medium by transferring the embryos to be imaged into a drop of culture medium.

21. Transfer the embryos into $500 \mu \mathrm{L}$ of culture medium in one well of an eight-well Lab-Tek II cover glass-bottomed dish.

Alternatively, image embryos in $\sim 300 \mu \mathrm{L}$ of culture medium in the depression created by the cover glass in MatTek dishes.

22. If imaging for $>8-10 \mathrm{~h}$, overlay the medium with embryo-tested mineral oil.

If using MatTek dishes, it is generally best to overlay the medium with mineral oil, even for short culture experiments.

23. Position the cover glass-bottomed dish on the microscope stage, ensuring that the local atmosphere is $5 \% \mathrm{CO}_{2}$ in air.

Make sure the dish has a lid on it; this will minimize both evaporation of medium and also embryo drift during imaging.

24. Select an objective for imaging. Set up the microscope control software to capture images or image volumes at the desired time interval.

Generally, 20X or 40X objectives are suitable for observing the movement of cells in the context of the entire embryo. A time-lapse interval of 8-15 min is a good compromise between good temporal resolution and minimizing photodamage to the embryo. Embryos can be imaged for $>12 \mathrm{~h}$ under such conditions.

See Troubleshooting.

\section{TROUBLESHOOTING}

Problem: The embryo fails to develop during imaging.

[Step 24]

Solution: This could be due to a number of reasons. Perform controls to determine the cause of the problem: 
1. Culture embryos in a tissue culture incubator.

i. If the embryos do not develop normally, there likely is a problem with one of the components of the culture medium. Try using freshly prepared medium with new components.

ii. Alternatively, small amounts of fixative or other noxious compounds could be compromising the culture. This can happen if, for example, tools used to manipulate embryos in fixative are used to isolate embryos for culture. Try keeping a set of tools exclusively for use with embryos for culture. If possible, isolate embryos in a location as clean as one used for tissue culture.

2. Culture embryos in the microscope without imaging. If the embryos do not develop, test the temperature stability within the enclosure. Use a commercially available temperature logger to record the temperature at preset intervals, and measure temperature fluctuations over the course of the culture period. Shifts in temperature below $37^{\circ} \mathrm{C}$ can be as bad for viability as fluctuations above $37^{\circ} \mathrm{C}$. A common cause for temperature fluctuations within the microscope enclosure is room airconditioning vents pointed directly at the microscope.

3. Test the $\mathrm{pH}$ of the culture medium to confirm that the local $\mathrm{CO}_{2}$ concentration is correct. If not, adjust the gas flow rate (it generally need not be high for a reasonably air-tight atmospheric enclosure).

4. Measure the volume of the culture medium before and after culture to determine whether the medium is evaporating. If it is, confirm that the gas is being humidified, or overlay the culture medium with mineral oil.

Problem: Embryos do not develop normally when imaged.

[Step 24]

Solution: This is indicative of photodamage to the embryo and can be solved by reducing the energy load on the embryo by one or more of the following methods:

1. Reduce the frequency of imaging by increasing the time lapse.

2. If you are taking image volumes, reduce the number of images in the stack.

3. Reduce the exposure time for each image.

4. Reduce the illumination intensity.

5. If you are using a scanning confocal microscope, reduce the pixel dwell time and/or the scan resolution, so that the total time the embryo is scanned is reduced.

6. All of the above approaches will lead to some degree of degradation of data quality. Loss of image brightness can be compensated for to some extent by increasing the gain or by binning. If fluorescence intensity does not need to be quantified and if using a detector that captures at $>8$-bit depth (i.e., >255 gray levels), one can use a histogram of the distribution of pixel intensities to adjust the exposure time to capture only 8 bits of information per channel, because this is a sufficient bit depth for images for figures. Where possible, use fluorophores that are excited at longer, lower energy wavelengths.

Problem: Embryos drift out of the field of view during imaging.

[Step 24]

Solution: This can be a difficult problem to avoid. Consider the following:

1. Temperature differences in the vicinity of the sample can set up convection currents in the culture medium. Make sure the environmental enclosure is well equilibrated.

2. The culture dish should be covered with a lid, even if the medium is overlaid with mineral oil. This generally reduces drift, presumably by preventing the incoming $\mathrm{CO}_{2}$ /air mix from causing eddies on the surface of the oil/culture medium that can shift the embryos.

3. If drift is still a problem and there is a risk of losing the embryo from the field of view altogether, try imaging with a lower magnification objective with a larger field of view. A 20X objective is generally a good compromise of magnification and field of view. 


\section{DISCUSSION}

\section{Pregastrulation Egg Cylinder Embryo}

Before gastrulation, the mouse egg cylinder consists of three tissues: the epiblast, the extraembryonic ectoderm, and the visceral endoderm, which encloses the first two. Although the epiblast gives rise to the majority of fetal tissues, a subset of visceral endoderm cells-the anterior visceral endoderm (AVE) — is responsible for correctly patterning the epiblast. The AVE arises at the distal tip of the egg cylinder and moves unidirectionally to a more proximal position, up to the boundary between the epiblast and extraembryonic ectoderm. It restricts the formation of the primitive streak to the opposite side of the epiblast, thereby inducing anterior neural characteristics in the underlying adjacent epiblast (for review, see Beddington and Robertson 1999; Srinivas 2006; Arnold and Robertson 2009; Rossant and Tam 2009). This movement fails to take place in mutants like Cripto (Ding et al. 1998), Otx2

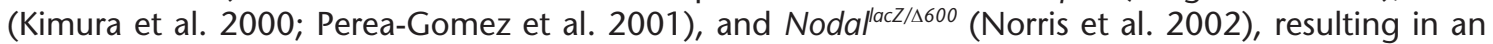
incorrectly positioned primitive streak and abnormal embryo development. The mechanism governing the translocation of AVE cells remains poorly understood, although time-lapse studies indicate that it is the result of active migratory movement (Srinivas et al. 2004).

There is significant cell movement in the epiblast of mouse embryos (Gardner and Cockroft 1998). Labeling studies in chick embryos suggest that the hypoblast (the equivalent of the mouse AVE) guides the movement of cells of the overlying epiblast (Foley et al. 2000). This raises the possibility that cells in the mouse epiblast, rather than moving at random, might do so in a directed manner. Better visualization of cell movements in the epiblast will help untangle this and other outstanding questions.

\section{Transgenic Mice for Imaging Egg-Cylinder Stage Embryos}

Cell movement in the surface visceral endoderm can be followed using bright-field illumination alone, but generally it is easier and more informative to follow specific cells that have been labeled fluorescently. Cells at this stage can be labeled using dyes such as Dil (Thomas et al. 1998), but there are an increasing variety of transgenic fluorescent reporter mice that make studies of cell movement at this stage easier because they provide embryos in which specific cell types are labeled by the expression of a fluorescent protein. Hex-GFP (Rodriguez et al. 2001) mice express green fluorescent protein (GFP) specifically in the AVE, and have been used in time-lapse imaging experiments (see Supplemental Data in Srinivas et al. 2004). Cerl-GFP (Mesnard et al. 2004), Hex-Venus, and Lefty-DsRed2 (Takaoka et al. 2006) transgenes have also been used to label the AVE. Lines that label the entire visceral endoderm include AFP-GFP (Kwon et al. 2006) and TTR-RFP (Kwon and Hadjantonakis 2009).

Mice expressing fluorescent reporters ubiquitously can also be used to visualize cells of the embryo at this stage. Several such lines exist, such as the CAG-TAG line (Trichas et al. 2008), which expresses a membrane-localized TdTomato and nuclear-localized enhanced green fluorescent protein (eGFP) bicistronically. The membrane label allows both movement and changes in cell shape to be followed over time (Fig. 3A). Another strategy useful in following cell movement is to use lines such as CAG-KikGR mice (Kurotaki et al. 2007), which express a photoconvertible protein ubiquitously. Cells can be "labeled" by photoconverting the green KikGR to red using 405-nm light (Fig. 3B). In addition to fluorescent lines, one can also use transgenic Cre driver lines in combination with fluorescent Cre reporters to label specific subsets of cells at this stage. For example, the MORE-Cre line (Tallquist and Soriano 2000) crossed with the R26R-eYFP reporter (Srinivas et al. 2001) labels epiblast cells in a "saltand-pepper" manner (resulting from the mosaic expression of the Cre), allowing one to follow the movement of individual epiblast cells (Fig. 3C).

The preceding brief discussion of transgenic lines is far from comprehensive and is meant only to illustrate some of the approaches possible for labeling cells. Many other useful lines exist, and new lines are being published regularly and can be found though various online resources.

\section{ACKNOWLEDGMENTS}

Rosa Beddington gave invaluable guidance in the culture of pregastrulation mouse embryos. I thank Kirstie Lawson for advice on the preparation of serum. I also thank the members of my group for their contributions, particularly Vivienne Wilkins for comments on the procedures, Tomoko Watanabe for capturing the photographs of embryo dissections, Georgios Trichas for the image of the CAGTAG embryo, and Bradley Joyce for the image of the KikGR embryo. Kohei Hatta very kindly shared the KikGR mice with us before publication. 


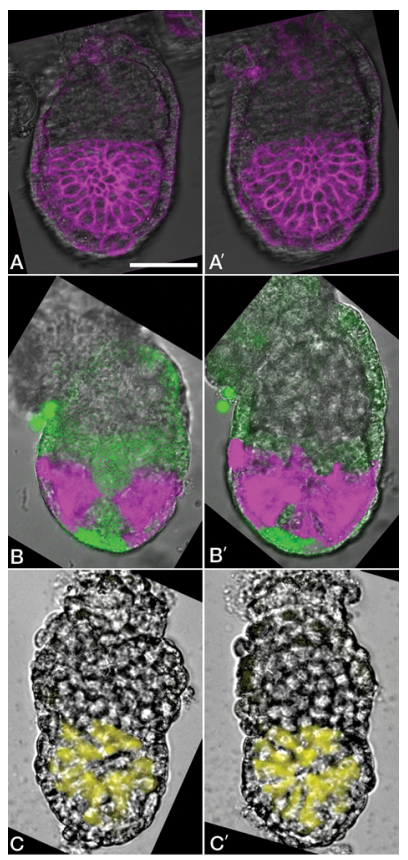

FIGURE 3. Examples of fluorescently labeled mouse embryos. In all three cases, the time between the first and the second image is $2 \mathrm{~h}$. $\left(A, A^{\prime}\right)$ A CAG-TAG embryo in which the epiblast and visceral endoderm is visualized by expression of a membrane-localized TdTomato (magenta). ( $\left.B, B^{\prime}\right)$ A KikGR embryo in which two regions of the epiblast have been labeled by photoconversion (magenta). The green fluorescence is the nonphotoconverted KikGR. $\left(C, C^{\prime}\right)$ An R26R-eYFP reporter embryo in which epiblast cells are labeled (yellow) through the mosaic expression of MORECre. Scale bar, $50 \mu \mathrm{m}$. (For color figure, see doi: 10.1101/pdb.prot5539 online at www.cshprotocols.org.)

\section{REFERENCES}

Arnold SJ, Robertson EJ. 2009. Making a commitment: Cell lineage allocation and axis patterning in the early mouse embryo. Nat Rev Mol Cell Biol 10: 91-103.

Beddington R. 1987. Isolation, culture, and manipulation of postimplantation mouse embryos. In Mammalian development: $A$ practical approach (ed. M Monk), pp. 43-69. IRL, Oxford, UK.

Beddington RS, Robertson EJ. 1999. Axis development and early asymmetry in mammals. Cell 96: 195-209.

Ding J, Yang L, Yan Y-T, Chen A, Desai N, Wynshaw-Boris A, Shen MM. 1998. Cripto is required for correct orientation of the anterior-posterior axis in the mouse embryo. Nature 395: 702-707.

Foley AC, Skromne I, Stern CD. 2000. Reconciling different models of forebrain induction and patterning: A dual role for the hypoblast. Development 127: 3839-3854.

Gardner RL, Cockroft DL. 1998. Complete dissipation of coherent clonal growth occurs before gastrulation in mouse epiblast. Development 125: 2397-2402.

Hogan B, Beddington R, Costantini F, Lacy E. 1994. Manipulating the mouse embryo: A laboratory manual, 2nd ed. Cold Spring Harbor Laboratory Press, Cold Spring Harbor, NY.

Kimura C, Yoshinaga K, Tian E, Suzuki M, Aizawa S, Matsuo I. 2000. Visceral endoderm mediates forebrain development by suppressing posteriorizing signals. Dev Biol 225: 304-321.

Kurotaki Y, Hatta K, Nakao K, Nabeshima Y, Fujimori T. 2007. Blastocyst axis is specified independently of early cell lineage but aligns with the ZP shape. Science 316: 719-723.

Kwon GS, Hadjantonakis A-K. 2009. Transthyretin mouse transgenes direct RFP expression or Cre-mediated recombination throughout the visceral endoderm. Genesis 47: 447-455.

Kwon GS, Fraser ST, Eakin GS, Mangano M, Isern J, Sahr KE, Hadjantonakis A-K, Baron MH. 2006. Tg(Afp-GFP) expression marks primitive and definitive endoderm lineages during mouse development. Dev Dyn 235: 2549-2558.

Mesnard D, Filipe M, Belo JA, Zernicka-Goetz M. 2004. The anteriorposterior axis emerges respecting the morphology of the mouse embryo that changes and aligns with the uterus before gastrulation. Curr Biol 14: 184-196.

Miura S, Mishina Y. 2003. Whole-embryo culture of E5.5 mouse embryos: Development to the gastrulation stage. Genesis
37: $38-43$.

Norris DP, Brennan J, Bikoff EK, Robertson EJ. 2002. The Foxh1dependent autoregulatory enhancer controls the level of Nodal signals in the mouse embryo. Development 129: 3455-3468.

Perea-Gomez A, Lawson KA, Rhinn M, Zakin L, Brûlet P, Mazan S, Ang S-L. 2001. Otx2 is required for visceral endoderm movement and for the restriction of posterior signals in the epiblast of the mouse embryo. Development 128: 753-765.

Rivera-Perez JA, Diefes H, Magnuson T. 2007. A simple enzymatic method for parietal yolk sac removal in early postimplantation mouse embryos. Dev Dyn 236: 489-493.

Rodriguez TA, Casey ES, Harland RM, Smith JC, Beddington RSP. 2001. Distinct enhancer elements control Hex expression during gastrulation and early organogenesis. Dev Biol 234: 304-316.

Rossant J, Tam PPL. 2009. Blastocyst lineage formation, early embryonic asymmetries and axis patterning in the mouse. Development 136: 701-713.

Srinivas S. 2006. The anterior visceral endoderm-Turning heads. Genesis 44: 565-572.

Srinivas S, Watanabe T, Lin C-S, William CM, Tanabe Y, Jessell TM, Costantini F. 2001. Cre reporter strains produced by targeted insertion of EYFP and ECFP into the ROSA26 locus. BMC Dev Biol 1: 4. doi: 10.1186/1471-213X-1-4.

Srinivas S, Rodriguez T, Clements M, Smith JC, Beddington RSP. 2004. Active cell migration drives the unilateral movements of the anterior visceral endoderm. Development 131: 1157-1164.

Takaoka K, Yamamoto M, Shiratori H, Meno C, Rossant J, Saijoh Y, Hamada H. 2006. The mouse embryo autonomously acquires anterior-posterior polarity at implantation. Dev Cell 10: 451-459.

Tallquist MD, Soriano P. 2000. Epiblast-restricted Cre expression in MORE mice: A tool to distinguish embryonic vs. extra-embryonic gene function. Genesis 26: 113-115.

Thomas PQ, Brown A, Beddington RS. 1998. Hex: A homeobox gene revealing peri-implantation asymmetry in the mouse embryo and an early transient marker of endothelial cell precursors. Development 125: 85-94.

Trichas G, Begbie J, Srinivas S. 2008. Use of the viral 2A peptide for bicistronic expression in transgenic mice. BMC Biol 6: 40 . doi: 10.1186/1741-7007-6-40. 


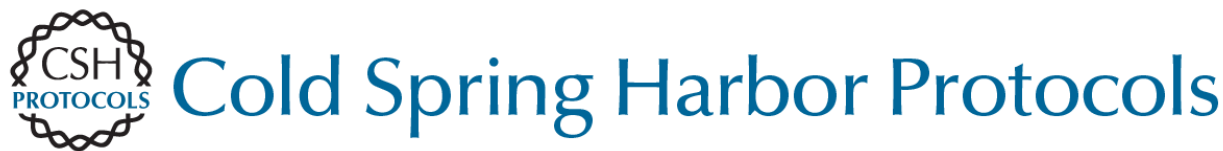

\section{Imaging Cell Movements in Egg-Cylinder Stage Mouse Embryos}

Shankar Srinivas

Cold Spring Harb Protoc; doi: 10.1101/pdb.prot5539

\begin{tabular}{|c|c|}
\hline $\begin{array}{r}\text { Email Alerting } \\
\text { Service }\end{array}$ & Receive free email alerts when new articles cite this article - click here. \\
\hline $\begin{array}{l}\text { Subject } \\
\text { Categories }\end{array}$ & $\begin{array}{l}\text { Browse articles on similar topics from Cold Spring Harbor Protocols. } \\
\text { Cell Biology, general (1382 articles) } \\
\text { Cell Imaging (525 articles) } \\
\text { Developmental Biology (728 articles) } \\
\text { Explant Culture (64 articles) } \\
\text { Imaging Development ( } 255 \text { articles) } \\
\text { Imaging/Microscopy, general (579 articles) } \\
\text { In Vivo Imaging (334 articles) } \\
\text { In Vivo Imaging, general (168 articles) } \\
\text { Laboratory Organisms, general (924 articles) } \\
\text { Mouse (437 articles) } \\
\text { Video Imaging / Time Lapse Imaging (171 articles) } \\
\text { Visualization (524 articles) } \\
\text { Visualization, general (369 articles) }\end{array}$ \\
\hline
\end{tabular}

